\title{
ETUdE DE LA CAVITATION PRODUITE PAR ETIRAGE DE BARRES DE ZIRCONIUM - APPLICATION DE LA DIFFUSION DES NEUTRONS AUX PETITS ANGLES -
}

\author{
A. R. dA COSTA*, M. PERnot*, C. SERVANT ot R. PENElle* \\ "GRECO "Grandes Deformations Endommagement" \\ Laboratoire de Metalurgle Structurale de l'Universite Paris-Sud, \\ U.A. 1107 du CNRS, 91405 Orsay Cedex, France
}

(Received October 1, 1985)

\section{INTRODUCTION}

Nous avons étudié la cavitation accompagnant la déformation plastique par étlrage de barres de zirconium polycristallines a l'alde de la technique de diffusion des neutrons aux petits angles (DNPA). Ces dernieres années, plusieurs travaux de recherche ont eté entrepris a l'aide de la DNPA, sur les phénomenes de cavitation se produisant solt sous Irradiation fortes doses $[1,2]$, solt au cours d'essals de fatigue a chaud $[3,4]$.

Par rapport a ces études, notre travall, classique dans sa démarche experimentaie dutilisation de la DNPA, presente par contre l'avantage de sulvre l'endommagement d'un materiau dont le mode de deformation correspond a une opération industrielle de $\mathrm{mise}$ en forme par etirage.

\section{METHODES EXPERIMENTALES}

Le zirconlum considere dans cette étude etalt de pureté nucléaire. Trois états differents par leur deformation ont eté soumls a l'analyse par DNPA. L'etat dit "Initlal" correspond a celul de barres de $50 \mathrm{~mm}$ de diemetre fliees a chaud. Le diametre de ces barres est ensulte amene par usinage a $25 \mathrm{~mm}$, puls par martelage a $18 \mathrm{~mm}$ (deformation equivalente: $\bar{\epsilon}=0,66$ ). Ces barres sublssent alors un recuit de recrlatallisation pendant in $650^{\circ} \mathrm{C}$. Une premiere réduction de diametre de 18 a $16,5 \mathrm{~mm}(\bar{\epsilon}=$ $0,17)$ par ettrage condult a l'état dit "intermediaire", une deuxieme réduction de 16,5 a $13 \mathrm{~mm}(\overline{\bar{z}}=$ $0,48)$ par fllage sous gaine d'acier inoxydable condult a l'état dit "final".

Les expérlences de DNPA ont eté realisees au Laboratoire Léon BRILLOUIN (CNRS-CEA, Saclay, France) et les resultats obtenus ont ete traltes a l'alde des programmes standards qui y sont disponibles. En utlisant deux tongueurs d'onde différentes $(\lambda=0,96 \mathrm{~nm}$ et $1,48 \mathrm{~nm})$, pour une distance echantillon-détecteur de 5 metres, les inconvénients dos a la diffraction multiple ont pu etre minimises. Le domalne de diffusion ainsi accessible est tel que $3.10^{-3} \mathrm{~nm}^{-1} \leqslant q \leqslant 5.10^{-1} \mathrm{~nm}^{-1}$ : q etant le vecteur de diffuslon défini par $q=4 \pi s i n \theta \cdot \lambda^{-1}$, ou $2 \theta$ est l'angle de diffusion. Les intensités diffusees ont eté enregistrees l'alde d'un detecteur bidimensionnel et ont eté transformees en section efficace differentlelle coherente (do/dn) par callbration par rapport au spectre de diffuslon de l'eau par l'equation de base sulvante:

dans Iaquelle:

$$
\frac{d \sigma}{d \Omega}(q)=\frac{\left(I_{E}-T_{E} I_{R}\right) T_{E}}{\left(I_{B_{2} O}-T_{B_{2} O} I_{C V}\right) T_{B_{2} O}} \cdot\left(\frac{d \sigma}{d R}\right)_{B_{2} O}
$$

- represente la section efflcace de diffusion coherente:

$\Omega \quad$ l'angle solide dans lequel s'opere la diffusion

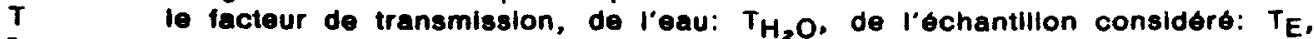

I l'intensite diffusee, par l'échantilion: $\mathrm{I}_{e}$, par l'eau: $\mathrm{I}_{2} \mathrm{O}$, par le dispositif experimental (y comprls le porte echantilion vide): IR, par la cellule d'eau de callbration vide: ICV. Nos echantillons avalent la forme de cylindres de $50 \mathrm{~mm}$ d'epalsseur et le volume de matière 
Irradie etait de $2208 \mathrm{~mm}^{3}$. Le diametre du faisceau ctant de $7,6 \mathrm{~mm}$. L'axe de révolution de ces cylindres, qui a place parallelement a la direction du laisceau de noutrons, coincidait avec la direction d'stirage du materlau.

\section{RESULTATS ET DISCUSSION}

Les intensites diffusees se présentalent distribuees de maniere lsotrope dans le plan d'observation, ce qui nous a permis de les regrouper et de les tralter a lialde des programmes lsotropes. Ce falt nous a donc suggéré d'utliser les modeles les plus simples consldérant des cavites de forme sphérlque. L'utllisation de l'approximation exponentielle de GUINIER [s], applicable en systeme dilue, aux courbes de diffusion obtenues l'alde des deux longueurs d'onde différentes, nous a permis:

- d'une part, de mettre en evidence l'existence d'une distributlon de la tallie des cavites quel que solt l'etat de detormation, car les polnts des dlagrammes de GUINIER (Fig. 1 et 2) ne sont pas allgnes sur des droltes:

- d'autre part, d'acceder avec la plus grande longueur d'onde $(\lambda=1,48 \mathrm{~nm})$ des cavites un peu plus grosses dans la dlatribution en tallie $\left(R_{g}=40 \mathrm{~nm}\right)$ que celle $\left(R_{g} \approx 30 \mathrm{~nm}\right)$ accesslbles avec $\lambda=0,96 \mathrm{~nm}$ (cl. Tableau $\mathrm{D}$, et dont la fraction volumique semble etre peu difierente comple tenu de la valeur extrapolée de $d \sigma / d \Omega$ (pour $q \rightarrow 0$ ) dans le cas des deux longueura d'onde.

Les dlametres des cavités ont été calcules en supposant des particules diffusantes uniformément distribuees dans le volume analyse. Dans cette hypothese le diametre des spheres correspondantes, $D$, vaut $2 R_{G}$ ( $R_{G}$ etant le rayon de GUINIER). $R_{G}=R g \sqrt{5 / 3}$ ou $R_{g}$ est le rayon de giration.

Tobleav

\begin{tabular}{|l|c|c|c|c|c|c|c|}
\hline Echantilion & $\bar{\varepsilon}$ & $\begin{array}{c}\mathrm{Rg}(\mathrm{nm}) \\
0,96 / 1,48\end{array}$ & $\begin{array}{c}\mathrm{D}(\mathrm{nm}) \\
0,96 / 1,48\end{array}$ & $\begin{array}{c}\Delta S / V\left(\mathrm{~nm}^{-1}\right) \\
0,96 / 1,48\end{array}$ & $\begin{array}{c}\mathrm{Rp}(\mathrm{nm}) \\
0,96 / 1,48\end{array}$ & $-\Delta d / \mathrm{d} .10^{-6}$ & $\begin{array}{c}\text { Df } \\
0,96 / 1,48\end{array}$ \\
\hline INITIAL & 0,66 & $32 / 41$ & $82 / 106$ & $344 / 183$ & $15 / 30$ & 3,73 & $2,34 / 2,48$ \\
INTERMED. & 0,17 & $32 / 40$ & $82 / 103$ & $852 / 341$ & $15 / 30$ & 114 & $2,27 / 2,28$ \\
FINAL & 0,48 & $30 / 42$ & $77 / 108$ & $1126 / 239$ & $15 / 30$ & 495 & $2,21 / 2,02$ \\
\hline
\end{tabular}

Afln de comparer ces résultats avec ceux obtenus par une autre approche de l'endommagement, nous avons effectue des observations en microscopie electronique a balayage sur l'etat flnal (ilg. 3). Les cavites qul sont detectes ont des talles de l'ordre de $0,25 \mu m$ a $3 \mu m$. II n'y a pas la contradiction mais confirmation du fait que la distribution en taille des cavites s'etale sur une tres large bande, les domaines de détection des deux techniques (DNPA et MEB) etant sensiblement adjacents avec peu ou pas de recouvrement.

. Dans la flgure 4, nous avons representé l'évolution de la surface totale des cavités diffusantes dans le volume irradie ( $\Delta S / V$ dans le Tableau I), pour les deux longueurs d'onde considéreses dans ce travall. Rappelons que $\Delta S / V$ est obtenu a partir de l'approximation asymptotlque de POROD [4] sulvant l'equation:

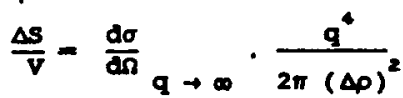

dans laquelle $\Delta p$ représente la difiérence de longueur de difiusion coherente entre le matérlau ( $\rho Z$ r $=$ $\left.0,71.10^{-12} \mathrm{~cm}\right)$ et le vide $(\rho=0)$. Pour $\lambda=0.96 \mathrm{~nm}$, nous constatons une augmentation de ( $\left.\Delta S / V\right)$ de l'etat Initial a l'etat final. Compte tenu que les rayons de POROD ( Tableau 1 ) des cavités diffusantes sont similaires, on peut conclure que le nombre de ces cavites augmente nettement avec le taux de deformation Imposé au cours des deux réductions de section des barres fllées.

Par contre pour $\lambda=1,48 \mathrm{~nm}$, on note que $(\Delta S / V)$ n'évolue pas de façon signiflcative. Dans le Tableau 1, nous avons porte les densites relatives des echantillons $(-\Delta d / d)$, on observe une chute importante de la densité. Donc sl l'on analyse simultanément tous les résultats, on note la présence de grosses cavites $(\sim 1 \mu \mathrm{m})$ "Invisibles" en DNPA, que la population de moyennes cavites $(\sim 0,1 \mu \mathrm{m})$ reste stable, et que la population de petites cavites $(<0,1 \mu \mathrm{m})$ augmente fortement, toutes ces cavites contribuant la chute de la densite. On peut donc supposer qu'll y a deux phénomenes: le grossissement des cavites (crolssance) et l'apparition de nouvelles cavites de petites tallies 
(germination).

Ces résultats sont en bon accord avec les conclusions de R. PAGE, J. R. WEERTMAN et M. ROTH [3] qui ont etudie la cavitation dans les Joints de grain du cuivre de haute purete, Induite lors d'essals de faligue effectues a haute temperature. Ces auteurs ont en effet montré, qu'en fonction du nombre crolssant de cycles, II se prodult d'une part un deplacement vers les diametres plus grands et d'autre part une nette augmentation du nombre de ces cavitos. Les rayons de GUINIER et de POROO restant respectivement pour les trols etats Identiques a $5 \%$ pres.

En appliquant la lol de POROD $(d \sigma / d \Omega)=C_{4} / q^{4}$ avec $C_{4}=$ constante de $\left.P O R O D\right)$, nous avons constate une variation de l'exposant theorlque -4 de cette lol, Flg, 5 et 6.

En effet, en passant de l'etat initial a l'etat final, la valeur de cet exposant evolue entre -3 et 4. Signalons que BOEUF el al. [ 6$]$, dans l'étude de la préclpltation des carbures $\mathrm{M}_{23} \mathrm{C}_{6}$ dans les aclers AISI 304, ont trouve une pente de $-3,7$ dans le graphe de POROD pour l'echantillon vielill a $700{ }^{\circ} \mathrm{C}$ pendant 5 jours. Ces auteurs ont conclu a une bonne verlfication de la lol de POROD (avec un exposant de -4) sans entrer dans le détail de la considération de la nettete de l'interface precipites-matrice sur le plan de la rugosite ou des fluctuations de la distribution des atomes. Alors qu'une variation semblable a celle obtenue sur le zirconlum a ete trouvee par H.D. BALE et P.W. SCHMIDT [7], dans l'etude de certalns charbons, du type llgnites. Ces auteurs ont propose d'expllquer ce falt a l'aide d'un modele qui considere la qualite de l'interface cavite-matrice. Dans cette nouvelle approche, $d \sigma / d \Omega=f(q-\alpha) o u=\alpha=$ ( 6 - Df) etant un coefficient dont les valeurs atteintes sont liees a la rugosite de l'Interface separant la particule diffusante de la matrice: cecl est tradult par les valeurs du coefficlent Df (dimension fractale). Pour Of $=2$, I'Interface est supposé lisse et on retrouve alors la valeur -4 de l'exposant de la lol de POROD.

Les figures 5 el 6 presentent les diagrammes de POROD, ainsi que les valeurs experimentales de l'exposant de la lol de POROD. Les valeurs de Df qui s'en déduisent sont portees dans le Tableau I. On constate dans les deux cas que Df se rapproche de 2 de l'etat initial vers l'etat flnal, donc que la qualite de l'interface s'amelioreralt statistlquement.

On peut emettre deux types d'hypotheses:

- soit les mecanismes de formation et de crolssance des cavites sont differents. Le premier correspondent a la creation d'une interface rugueuse a cause de la cristallographle de la detormation particullerement sélective dans le cas du zirconlum, cecl dans des zones de concentrations de contraintes: Interfaces precipite-matrice, Joints de grains, etc. . [8]. Le second provoquant un lissage statiatlque de l'interface. En effet, le caractere fractal est surfacique, alors que la crolssance et la coalescence Indulsent une reduction du rapport surface-volume.

- soit le mécanisme de germination évolue de telle façon que les cavités qui "naissent" solent de moins en molns fractales. Cette seconde hypothese pourrait correspondre au falt que les cavites qui "nalssent" en premier apparalssent sur des défauts (incompatiblities de déformation) alors qu'ensuite des cavites "nalssent" au milleu des grains.

Ces aspects sont approfondir par des observations en microscople électronique en transmission sur des répliques des surfaces des echantillons, alnsi que par des simulations d'evolutions de populations avec comme parametres les cinetiques de germination et de crolssance.

\section{REMERCIEMENTS}

Nous remercions vivement Mesdames A. BRULET, M. HENNION et $M$. B. FARNOUX du Laboratolre Leon Brillouin de Saclay d'avoir mis a notre disposition leur apparelliage de diffusion neutronlque aux petits angles et M. J.H. SCHMITT, chercheur au Laboratoire de Genle Physlque el Mecanique des Matérlaux de l'ENSIE de Grenoble, pour les mesures de densites relatives.

\section{REFERENCES}

[1] J.E. EPPERSON, R.W. HENDRICKS et K. FARREL, Philos. Mag. , (1974), 30, 803-817.

[2] C. HOFMEYR, K. ISEBECK ot R. M. MAYER, J. Appl. Cryst. (1975), 8, $193-194$.

[3] R. PAGE, J.R. WEERTMANN et M. ROTH, Acta Metall. (1982), 30, i357.

[4] M.H. YOO, J.C. OGLE, B.S. BORIE, E. H. LEE et R.W. HENDRICKS, Acta Metall. (1982) Vol. $30,1733-1742$.

[s] A. GUINIER, G. FOURNET, C. B. WALKER et K. L. YUDOWITCH, Smali-angle Scattering of X-rays (WIley, N. York 1955), P. 25, Eq (39).

[-] A. BOEUf, R.G.M. CACIUfFo, R. COPPOLA, S. CPICO, S. MELONE, P. PULITI, R. REBONATO ot F. RUSTICHELLI, J. Nucl. Mat., (1984) p. 9, 127.

[7] H.D. BALE et P.W. SCHMIDT, Phys. Rev. Letters, (1984) 53, 6, 596.

[*] S.H. GOODS et L.M. BROWN, Acta Metall. (1979) 27, 1-15. 

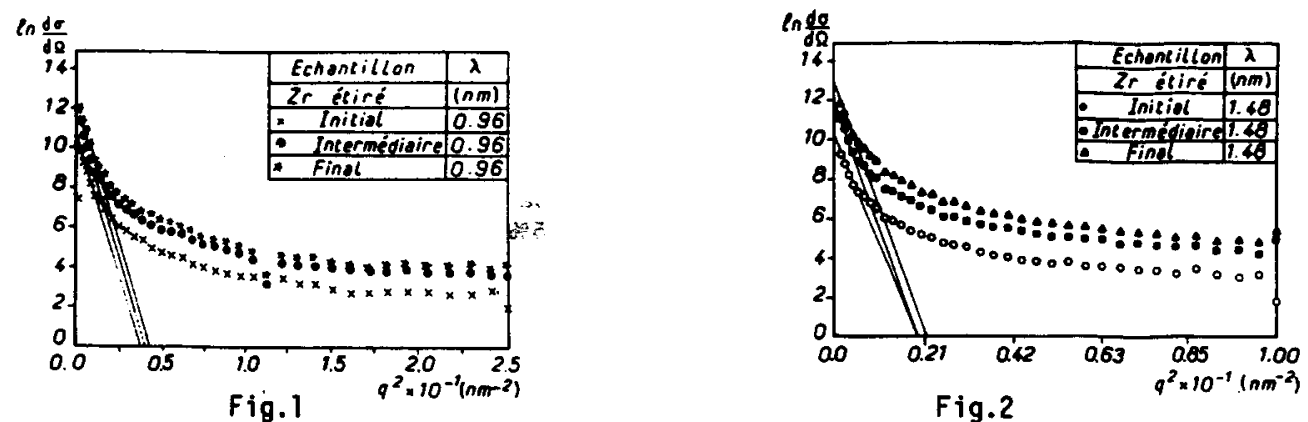

Diagrammes de GUINIER des résultats obtenus par DNPA pour les trois états déformés et les deux longueurs d'ondes utilisées.

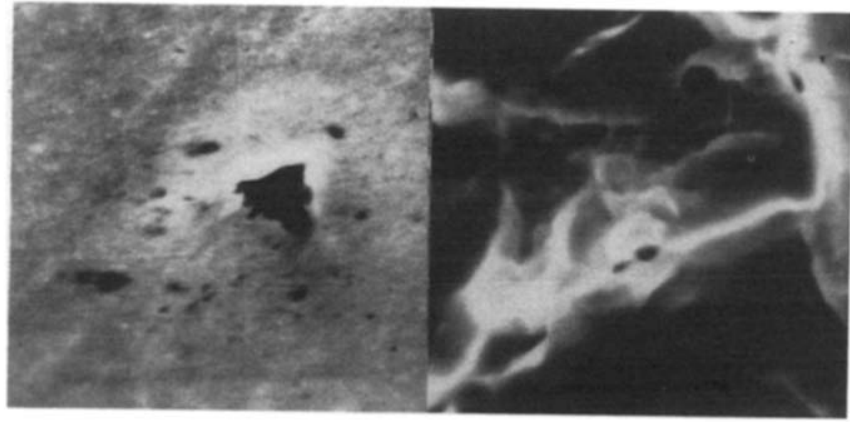

Fig.3 : Micrographies ẽlectroniques à balayage d'échantillons prélevés dans l'etat final ayant subi un polissage ionique (a), ayant été rompus ầ l'azote liquide (b).

(a)

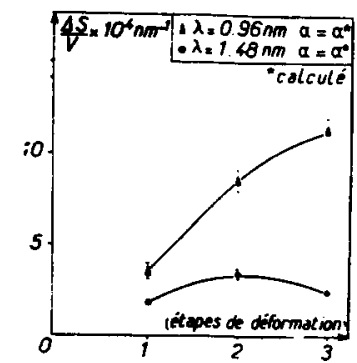

Fig.4: Graphes traduisant la variation de la surface spécifique des cavités diffusantes pour les trois ètats déformẻs et les deux $\lambda$ considérées.

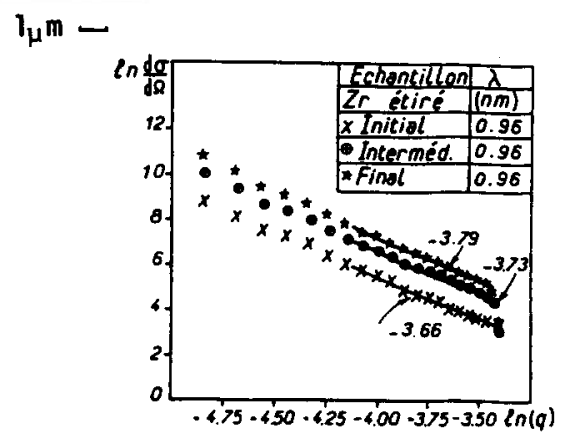

Fig.5 : Diagrammes de POROD des résultats obtenus par DNPA pour les trois états déformés étudiés avec $\lambda=0.96 \mathrm{~nm}$.

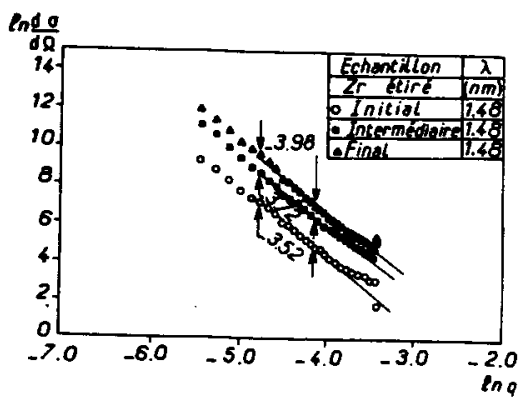

Fig. 6 :Diagrammes de POROD des résultats obtenus par DNPA pour les trois états déformés avec $\lambda=1.48 \mathrm{~nm}$. 